\title{
On spinless null propagation in five-dimensional space-times with approximate space-like Killing symmetry
}

\author{
Romulus Breban ${ }^{\mathrm{a}}$ \\ Institut Pasteur, 75724 Paris Cedex 15, France
}

Received: 7 July 2016 / Accepted: 19 August 2016 / Published online: 3 September 2016

(C) The Author(s) 2016. This article is published with open access at Springerlink.com

\begin{abstract}
Five-dimensional (5D) space-time symmetry greatly facilitates how a 4D observer perceives the propagation of a single spinless particle in a $5 \mathrm{D}$ space-time. In particular, if the 5D geometry is independent of the fifth coordinate then the $5 \mathrm{D}$ physics may be interpreted as $4 \mathrm{D}$ quantum mechanics. In this work we address the case where the symmetry is approximate, focusing on the case where the 5D geometry depends weakly on the fifth coordinate. We show that concepts developed for the case of exact symmetry approximately hold when other concepts such as decaying quantum states, resonant quantum scattering, and Stokes drag are adopted, as well. We briefly comment on the optical model of the nuclear interactions and Millikan's oil drop experiment.
\end{abstract}

\section{Introduction}

Geodesic propagation in five-dimensional (5D) space-times has remained, lately, a topic of great interest [1-8]. Particular attention has been devoted to 5D space-times, with a spacelike fifth dimension which is neither compact nor Planckian, where the metric is independent of the fifth dimension [7-10]. It has been shown that, in this case, 5D null-path propagation may be interpreted as anomaly-free 4D propagation both in the classical and quantum regimes $[7,8,11,12]$.

Why do we need thus a 5D formulation of physics? The approach to answer this question has been three fold.

First, studies of field equations (e.g., see [8-10]) and geodesic motion $[6,8]$ claimed that the existence of extra dimensions manifests as new physics in ordinary 4D spacetime. The relationship between geodesic motion and the classical tests of general relativity adapted for 5D space-times may be found in Ref. [8]. Furthermore, it has been postulated that phase experiments may carry a signature of the $5 \mathrm{D}$ nature of space-time [13].

a e-mail: breban@gmail.com
Second, it has been proposed that 5D space-time offers a unifying anthropic principle, since, inherently, we are 4D perceivers of Nature. In particular, it has been postulated that 5D symmetric space-times provide physical pictures depending on the symmetry $[11,12]$. Hence, if the 5D geometry is independent of the fifth coordinate then the 5D physics can be perceived as 4D quantum mechanics, while if the 5D geometry is independent of time then the 5D physics can be perceived as 4D statistical mechanics.

Third, studies began to address general 5D space-time geometries. A discussion of geodesic motion in non-symmetric 5D space-times is found in Ref. [6]. In this work we follow the same line of thought and address the case where symmetry is approximate. We postulate how concepts developed for the case where the symmetry is exact are used for the case where the symmetry is approximate. Of course, these concepts will work for a small region where the 5D spacetime appears to be symmetric. However, we show that they can be extended over larger regions if additional concepts such as decaying quantum states, resonant quantum scattering, and Stokes drag are adopted, as well. The price to pay for all these concepts working together is that general covariance no longer holds. We focus on the case where the 5D geometry depends weakly on the fifth coordinate. The case where the 5D geometry depends weakly on time leads to the statistical physics of time-dependent systems, near thermodynamical equilibrium. We defer this to further work.

The structure of the paper is as follows. In Sect. 2 we introduce our space-time geometry. In Sect. 3 we discuss quantum propagation using 5D null-path integrals. In Sect. 4 we discuss geodesic motion and then we conclude our work.

\section{Five-dimensional space-time}

We consider a 5D space-time with the metric $h_{A B}(A, B, \ldots$ $=0,1,2,3,5)$ containing one particle, and postulate that all 
quantum propagation of the particle takes place on $5 \mathrm{D}$ null paths [11]. A complete description of the propagation in the $5 \mathrm{D}$ space-time requires measurements of additional entities to $4 \mathrm{D}$ space-time events.

We discuss a special class of 5D space-times, foliated into conformally flat 4D space-times. The conformal factor is chosen to be the inverse square lapse of the foliation. The shift of the foliation, $N_{\mu} \equiv q A_{\mu} / c^{2}(\mu, v, \ldots=0,1,2,3)$, remnant of the $5 \mathrm{D}$ gravitational field, is interpreted as an external electromagnetic field by the 4D observer. The symbol $q$ stands for the specific charge of the particle-we use c.g.s. units throughout. In this context, $q / c^{2}$ represents a conversion constant between the gravitational and the electromagnetic field. The 5D field equations imply that $A_{\mu}$ (i.e., $N_{\mu}$ ) satisfy Maxwell-type equations [8-10].

We thus obtain a flat 4D space-time with superimposed electromagnetic field, fit for describing many experimental setups. We think of these space-times as local approximations of more realistic geometric constructs including non-trivial gravitational fields, whose metrics satisfy suitable field equations $[8,11]$. Removing the conformal factor from the metric since it is irrelevant for null-path counting, we have

$$
\begin{aligned}
& \tilde{h}_{A B}=\left(\begin{array}{cc}
\eta_{\mu \nu}+\frac{q^{2}}{c^{4}} A_{\mu} A_{v} & \frac{q}{c^{2}} A_{\mu} \\
\frac{q}{c^{2}} A_{v} & 1
\end{array}\right), \\
& \tilde{h}^{A B}=\left(\begin{array}{cc}
\eta^{\mu \nu} & -\frac{q}{c^{2}} A^{\mu} \\
-\frac{q}{c^{2}} A^{v} & 1+\frac{q^{2}}{c^{4}} A^{\rho} A_{\rho}
\end{array}\right),
\end{aligned}
$$

where the indices $\mu, v, \ldots$ are raised with $\eta^{\mu \nu}$.

\section{Path integrals}

Consider any two causally ordered events 1 and 2, with 1 in the past of 2 , which we write as $1 \prec 2$. Denote the coordinates of 1 and 2 by $x_{(1)}^{A}$ and $x_{(2)}^{A}$, respectively. Then the sum over $5 \mathrm{D}$ null paths between 1 and 2 , denoted here by $\mathcal{R}\left(x_{(1)}^{A}, x_{(2)}^{A}\right)$, is positively defined, is conformally invariant, and has the status of a microcanonical sum, determining the particle propagation between 1 and 2 [11]. The resulting 4D space-time is non-Lorentzian. The 5D infinitesimal null-path element $\mathrm{d} s_{5} \equiv \sqrt{\tilde{h}_{A B} \mathrm{~d} x^{A} \mathrm{~d} x^{B}}=0$ can be rewritten as

$\mathrm{d} s_{4 \pm} \equiv \mathrm{d} x^{5}= \pm \sqrt{-\eta_{\mu \nu} \mathrm{d} x^{\mu} \mathrm{d} x^{\nu}}-\frac{q}{c^{2}} A_{\rho} \mathrm{d} x^{\rho}$.

If $A_{\rho}$ is independent of $x^{5}$, then we obtain a 4D quantum mechanical picture [11]. $\mathrm{d} s_{4} \pm$ can be regarded as a 4D metric in a $4 \mathrm{D}$ non-Lorentzian curved manifold, where the distance is $\mathrm{d} s_{4} \pm$ if $1 \prec 2$, and $-\mathrm{d} s_{4} \pm$ if $2 \prec 1$. Computing the path integral $\mathcal{R}\left(x_{(1)}^{A}, x_{(2)}^{A}\right)$ in the 5D Lorentzian manifold with the metric $\tilde{h}_{A B}$ is equivalent to computing the following Feynman path integral [11]:
$\Psi_{ \pm}\left(\lambda^{-1} ; x_{(1)}^{\mu}, x_{(2)}^{\mu}\right)=\int\left[\mathrm{d}^{4} x\right] e^{i \lambda^{-1} s_{4} \pm(1 \prec 2)}$

of paths between $x_{(1)}^{\mu}$ and $x_{(2)}^{\mu}$, the 4D projections of $x_{(1)}^{A}$ and $x_{(2)}^{A}$, respectively, in a non-Lorentzian manifold. The symbol $\lambda$ represents the Compton wavelength of the propagating particle; i.e., $\lambda \equiv \hbar /(m c)$. It follows that computing the path integral $\mathcal{R}\left(x_{(2)}^{A}, x_{(1)}^{A}\right)$ is equivalent to computing $\Psi_{ \pm}^{*}\left(\lambda^{-1} ; x_{(1)}^{\mu}, x_{(2)}^{\mu}\right) . \Psi_{ \pm}$does not result as a scalar field on the 4D manifold, since a transformation of coordinates that reverses causality implies a complex conjugation of $\Psi_{ \pm}$.

If $A_{\rho}$ is not independent of $x^{5}$, then the traditional quantum mechanics picture no longer holds. The mass of the particle is no longer a constant of motion. We discuss a possible interpretation of the case where we have weak electromagnetic fields of the form $A_{\rho}+x^{5} A_{\rho}^{\prime}$ with $\partial_{5} A_{\rho}=0$ and $\partial_{5} A_{\rho}^{\prime}=0$. We obtain

$\mathrm{d} x^{5}= \pm \sqrt{-\eta_{\mu \nu} \mathrm{d} x^{\mu} \mathrm{d} x^{\nu}}-\frac{q}{c^{2}} A_{\rho} \mathrm{d} x^{\rho}-\frac{q}{c^{2}} x^{5} A_{\rho}^{\prime} \mathrm{d} x^{\rho}$.

Hence, it is not possible to naturally define a 4D path element. In fact, by separating the fifth coordinate and integrating, we obtain a non-local action,

$$
\begin{aligned}
s_{4 \pm}^{\prime}(1 \prec 2) \equiv & x_{(2)}^{5}-x_{(1)}^{5} \\
= & \int_{1 \prec 3 \prec 2}\left( \pm \sqrt{-\eta_{\mu \nu} \mathrm{d} x^{\mu} \mathrm{d} x^{\nu}}-\frac{q}{c^{2}} A_{\rho} \mathrm{d} x^{\rho}\right) \\
& \times \exp \left(\int_{3 \prec 2} \mathrm{~d} x^{\rho} \frac{q}{c^{2}} A_{\rho}^{\prime}\right),
\end{aligned}
$$

whose path integral

$\Psi_{ \pm}^{\prime}\left(\lambda^{-1} ; x_{(1)}^{\mu}, x_{(2)}^{\mu}\right)=\int_{1 \prec 2}\left[\mathrm{~d}^{4} x\right] e^{i \lambda^{-1} s_{4 \pm}^{\prime}(1 \prec 2)}$

satisfies, in the non-relativistic limit, the following differential equation obtained according to Feynman's procedure [14]:

$$
\begin{aligned}
& \pm \frac{\hbar}{i} \frac{\partial \Psi_{ \pm}^{\prime}}{\partial t} \\
& =\frac{1}{2 m}\left[\frac{\hbar}{i} \nabla-\frac{m q}{c} \vec{A}\right]^{2} \Psi_{ \pm}^{\prime} \mp m q A_{0} \Psi_{ \pm}^{\prime}+m c^{2} \Psi_{ \pm}^{\prime} \\
& \quad+\frac{\hbar}{i}\left(-\frac{c}{2} \frac{q}{c^{2}} A_{0}^{\prime} \pm \frac{q}{2 m c^{2}} \overrightarrow{A^{\prime}} \frac{\hbar}{i} \nabla \pm \frac{c}{2} \frac{q}{c^{2}} \vec{A} \frac{q}{c^{2}} \overrightarrow{A^{\prime}}\right) \Psi_{ \pm}^{\prime}
\end{aligned}
$$

$\Psi_{-}^{\prime}$ describes the forward propagation of a particle of mass $m$, while $\Psi_{+}^{\prime}$ describes the forward propagation of an antiparticle of mass $m$. Since the electromagnetic field depends on $x^{5}$, the mass is, in fact, no longer a constant of motion. However, the propagation is considered for a 4D particle of mass $m$, where terms in $A_{\rho}^{\prime}$ account for the change in the dynamics due to the change in mass. 
The term in round brackets is non-hermitian and problematic for an interpretation of quantum mechanics where probability is conserved. Still, non-hermitian hamiltonians are used to describe key processes such as decaying quantum states [15] and resonant quantum scattering [16]. In particular, we emphasize the success of the phenomenological model of nuclear interactions in the range of 10-100 MeV known as the optical model [17]. Summation over paths in space-times with complex coordinates, involving actions with complex potential, proved successful in further developing some of these theories [18]. Later, it was shown that paths in manifolds with real coordinates suffice [19]. Our approach uses both real interaction potentials and paths in manifolds with real coordinates.

\section{Geodesic propagation}

We discuss the classical limit where $\lambda \rightarrow 0$ and the propagation takes place on a single path where the action reaches a local extremum and which contributes most to the path integral. We denote by $\tau$ the parameter of a path and, using Eq. (5), write the lagrangian of the non-local action $s_{4 \pm}^{\prime}(1 \prec 2)$, with $1 \prec 3 \prec 2$, as follows:

$\mathcal{L} \equiv L+L^{\prime} \int_{1 \prec 4 \prec 3} \mathrm{~d} \tau L \exp \left(\int_{4 \prec 3} L^{\prime} \mathrm{d} \sigma\right)$,

where

$L= \pm \sqrt{-\eta_{\mu \nu} \dot{x}^{\mu} \dot{x}^{\nu}}-\frac{q}{c^{2}} A_{\rho} \dot{x}^{\rho}, \quad L^{\prime}=-\frac{q}{c^{2}} A_{\rho}^{\prime} \dot{x}^{\rho}$,

and $\dot{x}^{\mu} \equiv \mathrm{d} x^{\mu} / \mathrm{d} \tau$. Assuming that on the classical path

$\left|\int_{1 \prec 2} L^{\prime} \mathrm{d} \sigma\right| \ll 1$

we have ${ }^{1}$

$\mathcal{L} \approx L+L^{\prime} \int_{1 \prec 3} \mathrm{~d} \tau L$,

and the Euler-Lagrange equations are

$$
\begin{aligned}
& {\left[\frac{\mathrm{d}}{\mathrm{d} \tau}\left(\frac{\partial L}{\partial \dot{x}^{\mu}}\right)-\frac{\partial L}{\partial x^{\mu}}\right]} \\
& +\left[\frac{\mathrm{d}}{\mathrm{d} \tau}\left(\frac{\partial L^{\prime}}{\partial \dot{x}^{\mu}}\right) \int_{\tau_{1}}^{\tau} L d \sigma+\frac{\mathrm{d} L^{\prime}}{\mathrm{d} \tau} \int_{\tau_{1}}^{\tau} \frac{\partial L}{\partial x^{\mu}} \mathrm{d} \sigma-\frac{\partial L^{\prime}}{\partial x^{\mu}}\right. \\
& \left.\quad \times \int_{\tau_{1}}^{\tau} L \mathrm{~d} \sigma-L^{\prime} \int_{\tau_{1}}^{\tau} \frac{\partial L}{\partial x^{\mu}} \mathrm{d} \sigma+\frac{\partial L^{\prime}}{\partial \dot{x}^{\mu}} L+L^{\prime} \frac{\partial L}{\partial \dot{x}^{\mu}}\right]=0 .
\end{aligned}
$$

\footnotetext{
$\overline{1 \text { An alternate }}$ way to arrive at Eq. (11) is as follows. Equation (4), providing the infinitesimal action $\mathrm{d} x^{5}$ as a function of $x^{5}$, may be read as an equation for an attracting fixed point at $x^{5}$. Hence, for the first order in $L^{\prime}$, we perform one iteration toward the fixed point. That is, we choose $x_{(1)}^{5}=0$ and use $\mathrm{d} s_{4}(1 \prec 3)$ for a nested approximation of $\mathrm{d} s_{4 \pm}^{\prime}(1 \prec 2)$.
}

\subsection{A non-relativistic application}

To illustrate the 4D interpretation of 5D geodesic motion, we consider Eq. (12) under the following conditions. First, we assume a simple form of the electromagnetic potential,

$A_{\rho}+x^{5} A_{\rho}^{\prime}=(-z E, 0,0,0)+x^{5}\left(A_{0}^{\prime}, 0,0,0\right)$,

where $E$ and $A_{0}^{\prime}$ are constants. Second, the path parameter $\tau$ represents the proper time of the time-like geodesic in the 4D manifold with the metric $\eta_{\mu \nu}$; i.e., $\mathrm{d} \tau^{2}=-\eta_{\mu \nu} \mathrm{d} x^{\mu} \mathrm{d} x^{\nu}$. Third, we take the non-relativistic limit where $\tau \rightarrow c t$ and $\left|\mathrm{d} \dot{x}^{j} \mathrm{~d} \dot{x}_{j} / c^{2}\right| \ll 1$. Fourth, we multiply Eq. (12) by $m c .^{2}$ Hence, we obtain

$m \ddot{z}-\frac{m q}{c} A_{0}^{\prime} \dot{z} \pm(m q) E\left[1-\frac{q}{c} A_{0}^{\prime}\left(t_{2}-t_{1}\right)\right]=0$,

where we further neglect the term $\left|(q / c) A_{0}^{\prime}\left(t_{2}-t_{1}\right)\right|=$ $\left|\int_{t_{1}}^{t_{2}} L^{\prime} \mathrm{d} t\right| \ll 1$ [cf. Eq. (10)], meaning that the equation of motion holds for short time intervals $\left(t_{2}-t_{1}\right) \ll\left|c /\left(q A_{0}^{\prime}\right)\right|$. Hence, we arrive at

$m \ddot{z}-\frac{m q}{c} A_{0}^{\prime} \dot{z} \pm(m q) E=0$.

This corresponds to the 5D picture whereby a particle with momentum $p^{A}=(E / c, \vec{p}, m c)$ propagates with increasing mass, until eventually reaching the momentum $\hat{p}^{A}=$ $(E / c, 0, \hat{m} c)$, at rest. Equation (15) stands for the motion of a 4D particle in constant electric field, subject to a drag of Stokes type. In the case where $(m q) A_{0}^{\prime} / c<0$, the 4D particle with speed $\dot{z}$ brakes because of the interaction with a still environment. The case where $(m q) A_{0}^{\prime} / c>0$ is reduced to the previous case by a time reversal transformation. However, an interpretation by which the 4D particle is entrained by a moving environment is also possible.

The 5D setup with the electromagnetic potential given by Eq. (13) has yet another interpretation [11]. Since $A_{\rho}+$ $x^{5} A_{\rho}^{\prime}$ is time independent and motion is non-relativistic, the particle propagation may be described, as leading order, by the Langevin equation [11]

$m \ddot{z}+\zeta \dot{z} \pm(m q) E+\eta(t)=0$,

where $\zeta$ is the drag coefficient and $\eta(t)$ is a stochastic force with $\left\langle\eta(t) \eta\left(t^{\prime}\right)\right\rangle=2 \zeta k_{B} T \delta\left(t-t^{\prime}\right)$. In the limit where thermal fluctuations go to zero (i.e., $T \rightarrow 0$ ), the stochastic force vanishes. Hence, cf., Eqs. (15) and (16), we identify

\footnotetext{
${ }^{2}$ Because the translational symmetry along $x^{5}$ holds only approximately, $\lambda$ changes slowly with the proper time. We consider $\lambda$ as an approximate constant of motion. As a precaution for the physical interpretation, we use $\lambda^{-1} s_{4 \pm}^{\prime}$ rather than $s_{4 \pm}^{\prime}$ as physical action.
} 
- $(m q) A_{0}^{\prime} / c$ with $\zeta$. Furthermore, we rewrite the condition $\left|(q / c) A_{0}^{\prime}\left(t_{2}-t_{1}\right)\right|=\left|\int_{t_{1}}^{t_{2}} L^{\prime} \mathrm{d} t\right| \ll 1$ as $2\left(t_{2}-t_{1}\right) / u \ll 1$, where $u \equiv 2 m / \zeta$ is a quantum of physical time [11], defined for the case where $E=0$.

Particle motion described by Eqs. (15) and (16) was studied experimentally by Brown [20] and Millikan [21,22]. In particular, Millikan studied the motion on charged oil drops in constant electric field. By tuning $E$, while keeping everything else unchanged, one could in principle measure $m q$. However, to estimate $m q$, Millikan observed oil drops whose charge changed suddenly during the experiment, due to ionization. Hence, according to our theory, this corresponds to several single particle propagations. Explaining the experimental results obtained by Millikan requires additional physical principles to those described here.

\section{Discussion and conclusion}

Kaluza's ansatz to set up $\partial_{5}$ to zero leads to a unified formulation of field equation for the gravitational and electromagnetic fields [23]. However, even in the case where the metric is $x^{5}$-dependent, one may absorb the terms which contain $\partial_{5}$ into sources of the electromagnetic field. Hence, formally, a split of the 5D field equations between equations for the gravitational and the electromagnetic fields is possible even in the absence of the space-like symmetry. Therefore, we conclude that Maxwell-type equations, in agreement with experimental observations of electric fields, may be written even though the 5D space-time is not symmetric.

In previous work, we discussed an interpretation of a symmetric 5D space-time and introduced physical concepts such as mass and temperature to 4D geometry. In the case where the 5D space-time is approximatively symmetric, these concepts hold approximatively, in a small region of space-time. The description of the 5D geometry may be improved by using additional concepts such as decaying states, resonant quantum scattering, and Stokes drag. These concepts correct the previously proposed 4D picture [11] and extend the description of particle propagation for longer durations of proper time. Furthermore, we link newly obtained equations of motion to previously studied phenomenology. Hence, building on previous work, more and more 4D physics may be regarded from the perspective of a non-compact $5 \mathrm{D}$ spacetime.

Open Access This article is distributed under the terms of the Creative Commons Attribution 4.0 International License (http://creativecomm ons.org/licenses/by/4.0/), which permits unrestricted use, distribution, and reproduction in any medium, provided you give appropriate credit to the original author(s) and the source, provide a link to the Creative Commons license, and indicate if changes were made.

Funded by SCOAP ${ }^{3}$.

\section{References}

1. V. Diemer, J. Kunz, C. Lämmerzahl, S. Reimers, Dynamics of test particles in the general five-dimensional Myers-Perry spacetime. Phys. Rev. D 89(12), 124026 (2014)

2. J. Chandler, M.H Emam, Geodesic structure of five-dimensional nonasymptotically flat 2-branes. Phys. Rev. D 91(12), 125024 (2015)

3. S. Grunau, B. Khamesra, Geodesic motion in the (rotating) black string spacetime. Phys. Rev. D 87(12), 124019 (2013)

4. S. Grunau, V. Kagramanova, J. Kunz, Geodesic motion in the (charged) doubly spinning black ring spacetime. Phys. Rev. D 87(4), 044054 (2013)

5. S. Guha, P. Bhattacharya, S. Chakraborty, Particle motion in the field of a five-dimensional charged black hole. Astrophys. Space Sci. 341(2), 445-455 (2012)

6. J. Ponce de Leon, Equations of motion in Kaluza-Klein gravity reexamined. Gravit. Cosmol. 8, 272-284 (2002)

7. S.S. Seahra, P.S. Wesson, Null geodesics in five-dimensional manifolds. Gen. Relativ. Gravit. 33(10), 1731-1752 (2001)

8. P.S. Wesson, Space-time-matter. Modern Higher-dimensional Cosmology (World Scientific, 2007)

9. K. Kleidis, D. Papadopoulos, On the adiabatic expansion of the visible space in a higher-dimensional cosmology. Gen. Relativ. Gravit. 29(3), 275-290 (1997)

10. L. Faria-Busto, Some new cosmological results of quadratic lagrangians. Phys. Rev. D 38, 1741-1753, (1988). doi:10.1103/ PhysRevD.38.1741

11. R. Breban, Interpretation of the five dimensional quantum propagation of a spinless massless particle. Prog. Theor. Phys. 114(3), 643-668 (2005)

12. R. Breban, A five-dimensional perspective on the Klein-Gordon equation. Ann. Phys. 356, 158-170 (2015)

13. P.S. Wesson, Extra dimensions and phase experiments. Annalen der Physik 528(3-4), 307-312 (2015)

14. Richard P. Feynman, Space-time approach to non-relativistic quantum mechanics. Rev. Mod. Phys. 20, 367-387 (1948)

15. R.M. More, Theory of decaying states. Phys. Rev. A 4(5), 1782 $1790(1971)$

16. B. Buck, Calculation of elastic and inelastic proton scattering with a generalized optical model. Phys. Rev. 130(2), 712-726 (1963)

17. H. Feshbach, The optical model and its justification. Annu. Rev. Nucl. Sci. 8, 49-104 (1958)

18. T. Koeling, R.A. Malfliet, Semi-classical approximations to heavy ion scattering based on the Feynman path-integral method. Phys. Rep. 22(4), 181-213 (1975)

19. E.M. Wright, Path integral approach to the Schrödinger equation with a complex potential. Phys. Lett. A 104(3), 119-122 (1983)

20. R. Brown, The miscellaneous botanical works of Robert Brown, vol. 1 (R. Hardwicke, 1866)

21. R.A. Millikan, The isolation of an ion, a precision measurement of its charge, and the correction of Stokes's law. Phys. Rev. 32(4), 349 (1911)

22. R.A. Millikan, Brownian movements in gases at low pressures. Phys. Rev. 1(3), 218-221 (1913)

23. T. Kaluza. Zum Unitätsproblem der Physik. Sit. Preuss. Akad. Wiss. Phys. Mat. k1, 966 (1921) 